\title{
DESIGN OF 3D RC FRAME ON SLOPING GROUND
}

\author{
Shivanand.B ${ }^{1}$, H.S.Vidyadhara ${ }^{2}$ \\ ${ }^{1}$ P.G.Student, Department of Civil Engineering, Poojya Doddappa Appa college of Engineering, Gulbarga, 585102 \\ ${ }^{2}$ Associate Professor, Department of Civil Engineering, Poojya Doddappa Appa college of Engineering, Gulbarga, \\ 585102
}

\begin{abstract}
The buildings resting on hill areas have to be configured differently from flat ground. Hill buildings are different from those in plains; they are very irregular and unsymmetrical in horizontal and vertical planes, and torsionally coupled \& hence susceptible to sever damage when affected by earthquake. The floors of such buildings have step back towards the hill slope and at the same time setback also. In this study 3D analytical model of 12 storied building have been generated for symmetric and asymmetric case. Building models are analyzed and designed by ETABS software to study the effect of influence of bracings, shear wall at different positions. Seismic analysis done by linear static (ESA), linear dynamic (RSA) and non-linear static Analysis (Pushover Analysis)
\end{abstract}

Keywords: Earthquake, Sloping Ground, ETABS, Pushover analysis. - ***

\section{INTRODUCTION}

Earthquakes in many parts of the world have concerned the issue regarding the safety of existing buildings. The present buildings, which were designed and constructed according to earlier code provisions, do not satisfy requirements of current seismic code and design practices. Therefore it is essential to safe unacceptable hazards to property and life of occupants, posed during future expecting earthquake. The safety of hazards is possible by means of seismic evaluation and performance, retrofitting of inadequate existing building structures. Framed buildings are getting pace in sloped areas particularly in hills, because of increased population and the land value. And thus, many of them are constructed on slopes and curved grounds.

Multistoried buildings on sloping ground are infrequent over level grounds whereas on hilly slopes these are quite common. Disaster due to Earthquake has always been one of the greatest natural calamities thrust upon the mankind since time immemorial and bringing in its wake untold miseries and hardships to the people affected. The economic growth $\&$ rapid urbanization in sloping region has accelerated the real estate development .due to this population density in hilly region has increased enormously. Therefore there is popular \& pressing demand for the construction of multistoried on sloping ground in around the cities.

Hence, they are susceptible to severe damage when affected by earthquake ground motion. Past earthquakes [e.g. Kangra (1905), Bihar- Nepal (1934 \& 1980), Assam (1950), Tokachi-Oki-Japan (1968), Uttarkashi-India (1991)][1], have proved that buildings located near the edge of stretch of hills or sloping ground suffered severe damages. Such buildings have mass and stiffness varying along the vertical and horizontal planes, resulting the center of mass and center of rigidity do not coincide on various floors. This requires torsional analysis; in addition to lateral forces under the action of earthquakes.

\subsection{Bracings}

Bracing systems are used to resist horizontal forces(wind load, seismic action) and to transmit to the foundation. The bracing members are arranged in many forms, which carry solely tension, or alternatively tension and compression. The bracing is made up of crossed diagonals, when it is designed to resist only tension. Based on the direction of wind, one diagonal takes all the tension while the other diagonal is assumed to remain inactive . one of the most common arrangements is the cross bracing. Bracings hold the structure stable by transferring the loads sideways (not gravity, but wind or earthquake loads) down to the ground and are used to resist lateral loads, thereby preventing sway of the structure.

\subsection{Shear Wall}

The usefulness of the shear wall in structural planning of multistory building has long been recognized. When walls are situated in the advantageous position in a building,they can very efficient in resisting lateral load on building, if not the whole amount \& the horizontal shear force originated from the load, are offend assign to such structural elements ,they have been called shear wall. The uses of shear walls or their equivalent become impressive in certain high rise structure if inter story deflections, caused by lateral loading are to be controlled. Well design shear walls in seismic areas have a very good performance.

\section{MODEL DISCREPTION}

Basically model consists of 3 bays with 12 storey building, each bay having a dimension of $7 \mathrm{~m}$ in $\mathrm{X}$ direction and $5 \mathrm{~m}$ in $\mathrm{Y}$ direction. The story height is kept $3.5 \mathrm{~m}$ and foundation height as $1.5 \mathrm{~m}$ below ground level. Beam size is of 
$0.3 \mathrm{mX} 0.6 \mathrm{~m}$ and Column size is of $0.7 \mathrm{mX0.85m}$. Slab Thickness is of $0.12 \mathrm{~m}$. The models are analyzed on sloping

ground as well as level ground.

\subsection{Models under Study}

\subsubsection{Set 1: Step Back Buildings on Sloping Ground}

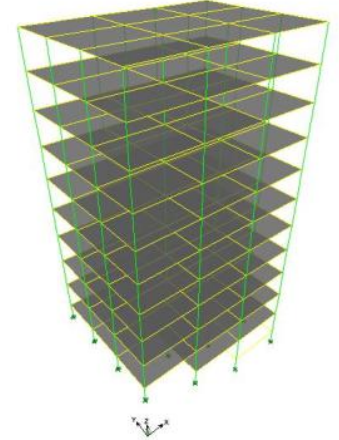

Fig:1

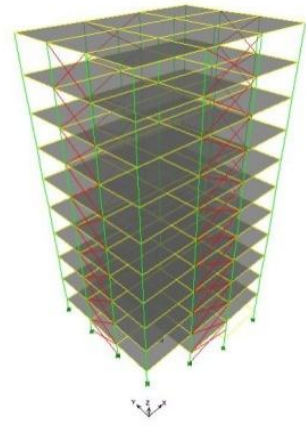

Fig:2

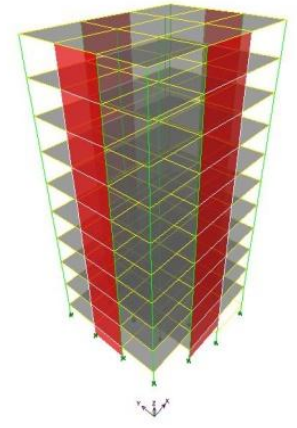

Fig:3

Model 1(M-1): Set Back bare frame. However, masses of wall are included Fig1

Model 2(M-2): Set Back frame has RC X bracings in outrigger patterns in the middle stories in all four sides Fig2

Model 3(M-3): Set Back frame has RC Shear Wall bracings in outrigger patterns in the middle stories in all four sides Fig 3

\subsubsection{Set 2: Step Back-Step Back Buildings on Sloping Ground}

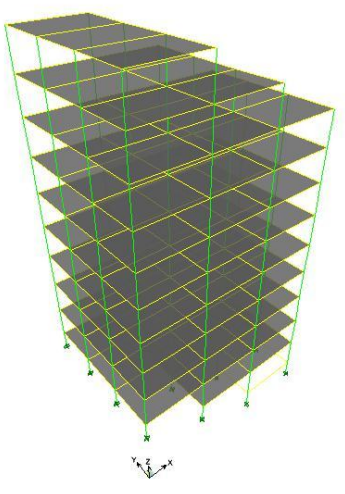

Fig: 4

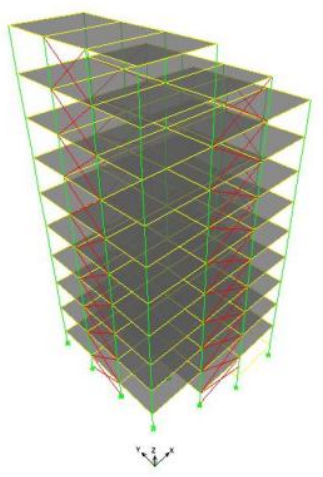

Fig: 5

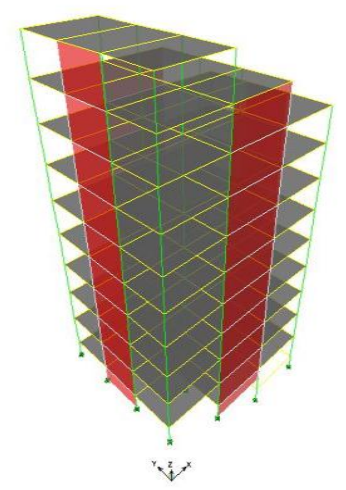

Fig: 6

Model 4(M-4): SetbackStepback bare frame. However, masses of wall are included. Fig 4

Model 5(M-5): SetbackStepback frame has RC X bracings in outrigger patterns in the middle stories all four sides. Fig 5

Model 6(M-6): SetbackStepback frame has RC Shear Wall bracings in outrigger patterns in the middle stories all four sides. Fig 6

\subsubsection{Set 3: Buildings on Plain Ground}

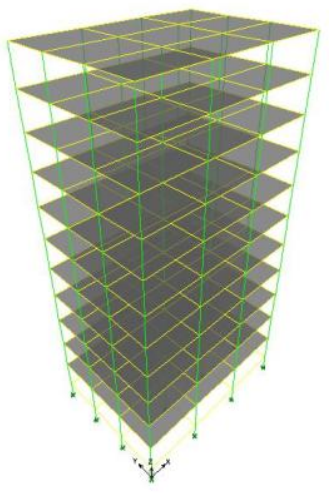

Fig: 7

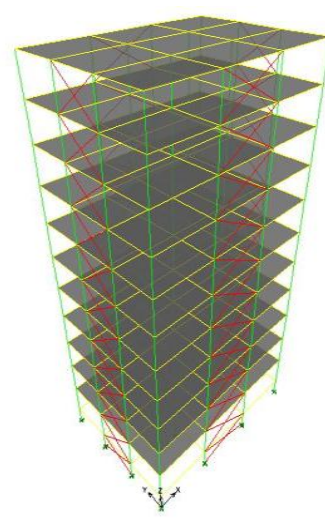

Fig: 8

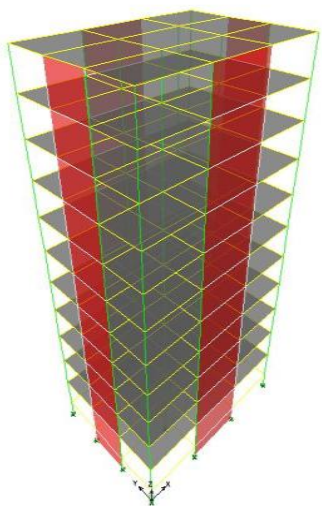

Fig: 9 
Model 7(M-7): Building modeled as bare frame. However, masses of the walls are included. Fig 7

Model 8(M-8): Building modeled has RC X bracings in outrigger patterns in the middle stories all four sides. Fig 8

Model 9(M-9): Building modeled has Shear wall in outrigger patterns in the middle stories all four sides. Fig

\section{RESULTS \& DISCUSSION}

Table: 1 Fundamental Time Period (Sec)

\begin{tabular}{|c|c|c|c|c|}
\hline & \multicolumn{2}{|c|}{ IS Code 1893-2002 } & \multicolumn{2}{|c|}{ ETABS Analysis } \\
\hline Model & Longitudinal & Transverse & Longitudinal & Transverse \\
\hline & & & \multicolumn{2}{|c|}{ Sloping Ground } \\
\hline & & & \multicolumn{2}{|c|}{ Set:1(Step Back) } \\
\hline M-1 & 1.134 & 1.134 & 1.69 & 1.69 \\
\hline M-2 & 1.134 & 1.134 & 1.24 & 1.24 \\
\hline \multirow[t]{2}{*}{ M-3 } & 1.134 & 1.134 & 0.958 & 0.958 \\
\hline & & & \multicolumn{2}{|c|}{ Set:2(SetbackStepback) } \\
\hline M-4 & 1.134 & 1.134 & 1.535 & 1.535 \\
\hline M-5 & 1.134 & 1.134 & 1.114 & 1.114 \\
\hline \multirow[t]{2}{*}{ M-6 } & 1.134 & 1.134 & 0.839 & 0.839 \\
\hline & & & \multicolumn{2}{|c|}{ Set:3 Plain Ground } \\
\hline M-7 & 1.134 & 1.134 & 2.075 & 2.075 \\
\hline M-8 & 1.134 & 1.134 & 1.309 & 1.309 \\
\hline M-9 & 1.134 & 1.134 & 1.117 & 1.117 \\
\hline
\end{tabular}

The codal (IS1893-2002) and analytical natural periods of the building models in longitudinal and transverse direction are shown in table 1 .From table it is apparent that the time periods obtained by the codal and modal analysis, do not agree, where the percentage difference of the fundamental periods between the codal and modal methods is maximum for model-1 \& model-7 of Sloping Ground \& Plain Ground respectively both along longitudinal $\&$ transverse direction.

It can be observed that from the tables ,the natural time periods for models along longitudinal \& transverse direction is reduced by $26.6 \%, 43.4 \%$, for model-2,model-3 \& respectively for step back buildings resting on sloping ground.

The natural time periods for models along longitudinal \& transverse direction is reduced by $27.36 \%, 45.3 \%$, for model-5, model-6 \& respectively for StepBackSetBack buildings resting on sloping ground.

The natural time periods for models along longitudinal \& transverse direction is reduced by $36 \%, 46.3 \%$, for model- 8 , model-7 \& respectively for buildings resting on Plain ground.

It can be observed that the in the M-6 presence of bracings, shear wall significantly affects the fundamental periods of vibration, which is a function of stiffness mass and damping characteristics of the building.

\subsection{Lateral Displacement:}

The Maximum displacement at each floor level with respect to ground are shown in Table: 2 to 13 for ESA \& RSA For better compatibility the displacement for each model along both direction of ground motion are plotted in graphs as shown from Fig 15 to 21

\section{Set 1(Step Back Buildings)}

In ESA it has been found that step back buildings M-2 ,M-3 has $57.3 \%$ \& $71.7 \%$ respectively less displacement compared to M-1 in Longitudinal direction \& in Transverse direction M-2,M-3 has $53.6 \%$ \& $63.9 \%$ respectively less displacement compared to M-1.

\section{Set2 (SetbackStepback)}

In ESA it has been found that the SetbackStepback buildings M-5 ,M-6 has $52.8 \%, 62.8 \%$ respectively less compared to M-4 in Longitudinal Direction \& In Transverse direction M5 ,M-6 has 50.2\%,59.7\% respectively less Compared to M4.

\section{Set 3(Plain Ground)}

In ESA it has found that the building resting on normal ground M-8,M-9 has $62.9 \%$ \& $74.4 \%$ respectively less compared to M-7 in Longitudinal direction \& in Transverse direction M-8,M-9 has $50.3 \%$ \& $59.7 \%$ respectively less compared to M-7.

\section{Set1 (Step Back Buildings)}

In RSA it can be seen that the M-2,M-3 has $27.8 \%$ \& $44.05 \%$ respectively less in Longitudinal direction \& in Transverse direction M-2,M-3 has $22.95 \%$ \& $40.6 \%$ respectively less compared to $\mathrm{M}-1$. 
Set2 (SetbackStepback Buildings)

In RSA it can be seen that the M-5,M-6 has 27\%,42.8\% respectively less in Longitudinal direction \& in Transverse direction M-5,M-6 has $30.3 \%, 46.7 \%$ respectively less compared to M-7.

\section{Set 3(Plain Ground)}

In RSA it can be seen that M-8, M-9 has $37.7 \%$ \& $47.9 \%$ respectively less in longitudinal direction $\&$ in Transverse direction M-8, M-9 has 30.3\%, 46.7\% respectively less compared to $\mathrm{M}-7$

From the results it is clear that the Set 2 type SetbackStepback buildings resting sloping ground produce less displacement compared to of Set1 Step back Buildings, $\&$ the Presence of Bracings, Shear Wall reduces the lateral displacement considerably both by ESA \& RSA.

Table 2: Lateral displacement in X-direction (ESA)

\begin{tabular}{|l|l|l|l|}
\hline \multicolumn{5}{|l|}{ step back } & M-2 & M-3 \\
\hline Story & M-1 & M-2 & 20.51 \\
\hline 12 & 72.477 & 30.9 & 18.33 \\
\hline 11 & 69.07 & 29.144 & 16.02 \\
\hline 10 & 64.147 & 26.779 & 13.64 \\
\hline 9 & 57.509 & 23.819 & 11.23 \\
\hline 8 & 49.315 & 20.374 & 8.848 \\
\hline 7 & 39.888 & 16.574 & 6.554 \\
\hline 6 & 29.656 & 12.552 & 4.427 \\
\hline 5 & 19.21 & 8.4535 & 2.553 \\
\hline 4 & 9.4787 & 4.4981 & 1.008 \\
\hline 3 & 2.1763 & 1.2381 & 0 \\
\hline 2 & 0 & 0 & 0 \\
\hline 1 & 0 & 0 & \\
\hline
\end{tabular}

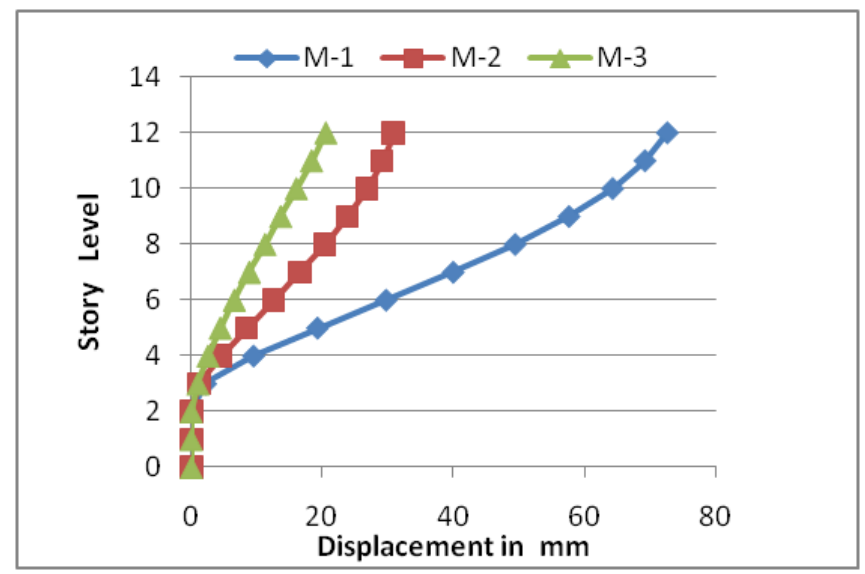

Fig 10: Lateral displacement in X-direction
Table 3: Lateral displacement in Y- Direction (ESA)

\begin{tabular}{|l|l|l|l|}
\hline \multicolumn{5}{|l|}{ Step Back } & \multicolumn{5}{l|}{} \\
\hline Story & M-1 & M-2 & M-3 \\
\hline 12 & 60.96 & 28.285 & 22 \\
\hline 11 & 58.45 & 26.616 & 19.65 \\
\hline 10 & 54.51 & 24.338 & 17.1 \\
\hline 9 & 49.05 & 21.525 & 14.41 \\
\hline 8 & 42.26 & 18.3 & 11.64 \\
\hline 7 & 34.44 & 14.79 & 8.88 \\
\hline 6 & 25.9 & 11.125 & 6.236 \\
\hline 5 & 17.03 & 7.4405 & 3.842 \\
\hline 4 & 8.497 & 3.9014 & 1.847 \\
\hline 3 & 1.831 & 0.9558 & 0.432 \\
\hline 2 & 0 & 0 & 0 \\
\hline 1 & 0 & 0 & 0 \\
\hline
\end{tabular}

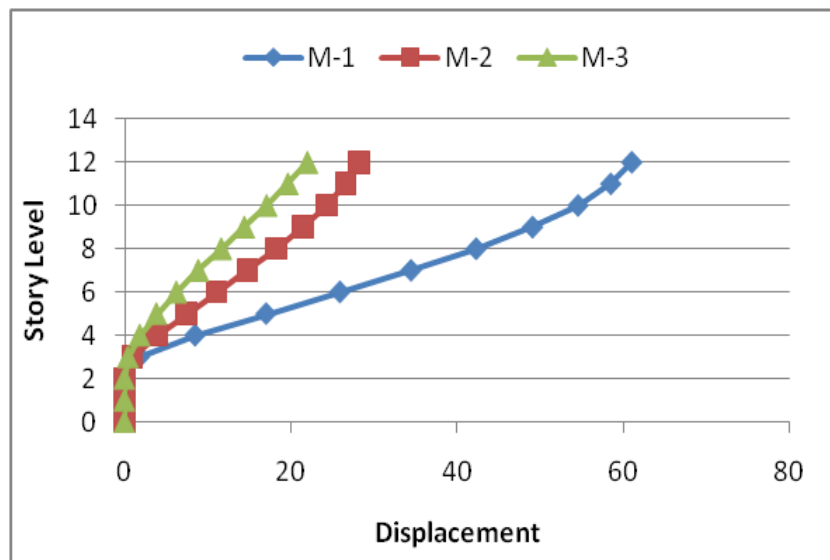

Fig 11: Lateral displacement in Y-direction

Table 4: Lateral displacement in longitudinal direction (ESA)

\begin{tabular}{|c|c|c|c|}
\hline \multicolumn{4}{|c|}{ SetBack StepBack } \\
\hline Story & M-4 & M-5 & M-6 \\
\hline 12 & 63.714 & 30.012 & 23.65 \\
\hline 11 & 60.508 & 28.238 & 21.35 \\
\hline 10 & 56.294 & 26.054 & 18.7 \\
\hline 9 & 50.816 & 23.401 & 15.99 \\
\hline 8 & 43.947 & 20.209 & 13.21 \\
\hline 7 & 35.824 & 16.571 & 10.45 \\
\hline 6 & 26.808 & 12.63 & 7.766 \\
\hline 5 & 17.453 & 8.5484 & 5.265 \\
\hline 4 & 8.6456 & 4.5673 & 3.049 \\
\hline 3 & 1.9925 & 1.2631 & 1.21 \\
\hline 2 & 0 & 0 & 0 \\
\hline 1 & 0 & 0 & 0 \\
\hline
\end{tabular}




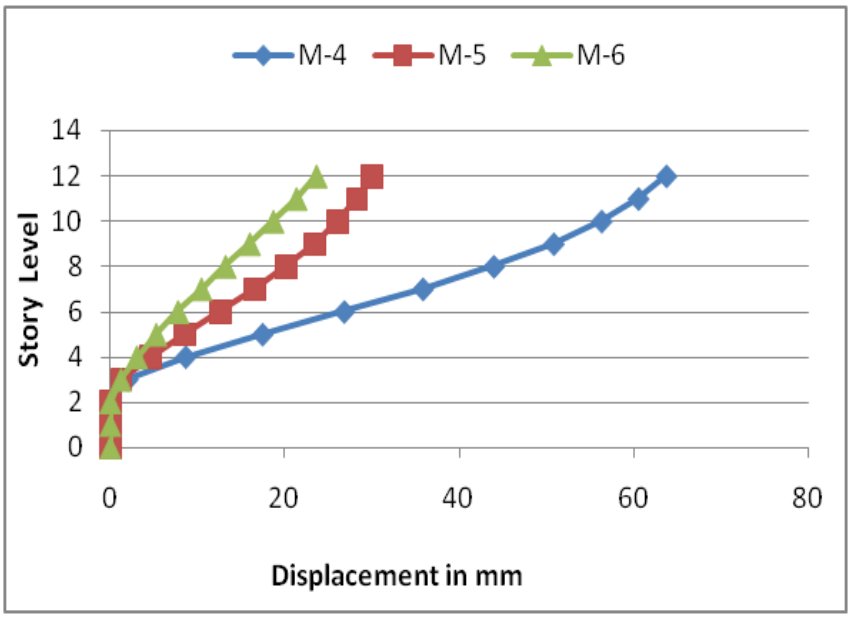

Fig 12: Lateral displacement in longitudinal direction

Table 5: Lateral displacement in Transverse direction (ESA)

\begin{tabular}{|l|l|l|l|}
\hline \multicolumn{4}{|l|}{ SetbackStepback } \\
\hline Story & M-4 & M-5 & M-6 \\
\hline 12 & 62.22 & 30.969 & 25.07 \\
\hline 11 & 59.85 & 29.25 & 22.41 \\
\hline 10 & 56.06 & 26.917 & 19.57 \\
\hline 9 & 50.67 & 23.959 & 16.55 \\
\hline 8 & 43.82 & 20.482 & 13.41 \\
\hline 7 & 35.8 & 16.626 & 10.26 \\
\hline 6 & 26.95 & 12.547 & 7.223 \\
\hline 5 & 17.7 & 8.4065 & 4.459 \\
\hline 4 & 8.778 & 4.402 & 2.146 \\
\hline 3 & 1.821 & 1.0531 & 0.497 \\
\hline 2 & 0 & 0 & 0 \\
\hline 1 & 0 & 0 & 0 \\
\hline
\end{tabular}

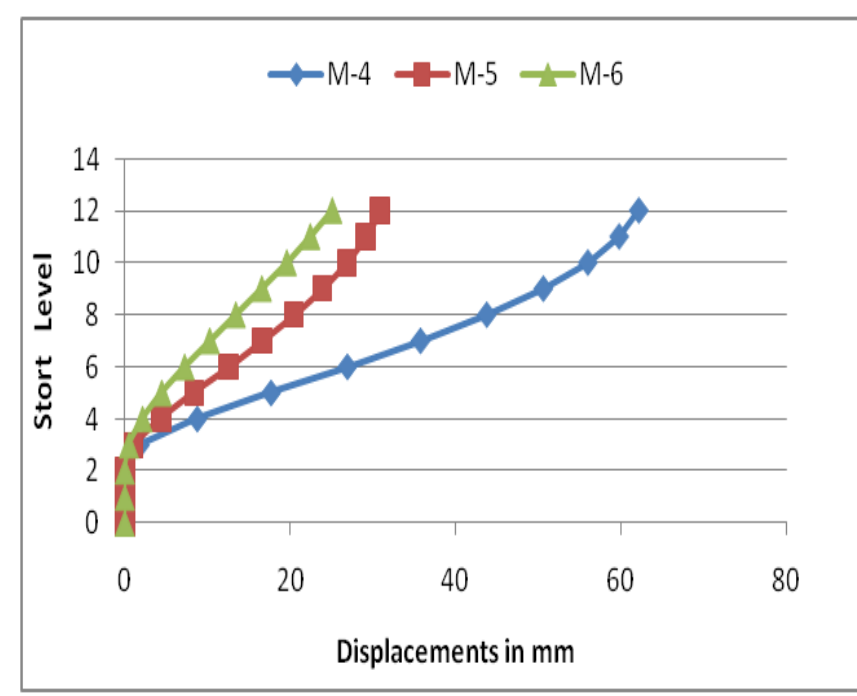

Fig 13: Lateral displacement in Transverse direction
Table 6: Lateral displacement in Longitudinal -direction (ESA)

\begin{tabular}{|l|l|l|l|}
\hline \multicolumn{4}{|l|}{ Plain Ground } \\
\hline Story & M-7 & M-8 & M-9 \\
\hline 12 & 102.96 & 38.193 & 26.32 \\
\hline 11 & 99.214 & 35.098 & 23.74 \\
\hline 10 & 93.835 & 31.632 & 21.02 \\
\hline 9 & 86.596 & 27.914 & 18.22 \\
\hline 8 & 77.644 & 24.022 & 15.38 \\
\hline 7 & 67.273 & 20.047 & 12.55 \\
\hline 6 & 55.829 & 16.092 & 9.795 \\
\hline 5 & 43.687 & 12.264 & 7.202 \\
\hline 4 & 31.286 & 8.6775 & 4.854 \\
\hline 3 & 19.227 & 5.4522 & 2.844 \\
\hline 2 & 8.5284 & 2.6859 & 1.267 \\
\hline 1 & 1.1851 & 0.522 & 0.202 \\
\hline
\end{tabular}

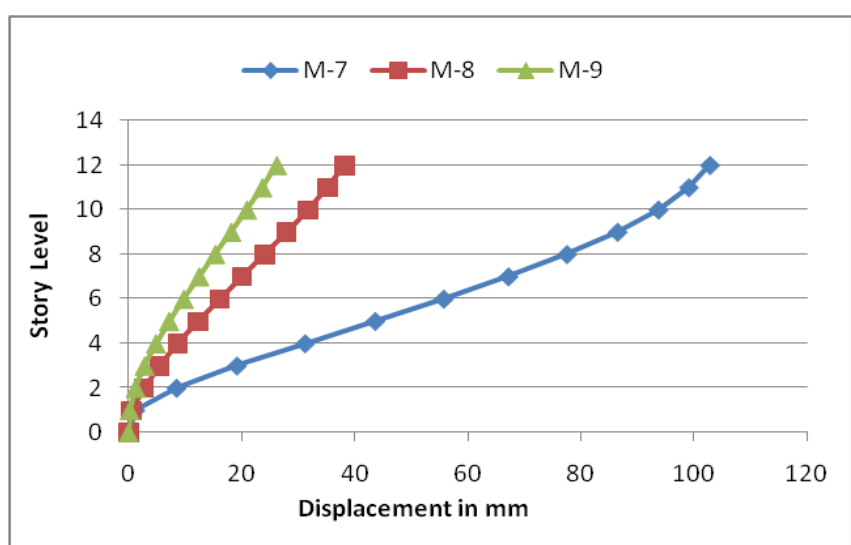

Fig 14: Lateral displacement in Longitudinal direction

Table 7: Lateral displacement in Transverse direction (ESA)

\begin{tabular}{|c|c|c|c|}
\hline \multicolumn{4}{|c|}{ Plain Ground } \\
\hline Story & M-7 & M-8 & M-9 \\
\hline 12 & 87.04 & 44.943 & 35.39 \\
\hline 11 & 84.17 & 41.319 & 32.21 \\
\hline 10 & 79.78 & 37.255 & 28.8 \\
\hline 9 & 73.74 & 32.897 & 25.2 \\
\hline 8 & 66.28 & 28.314 & 21.47 \\
\hline 7 & 57.68 & 23.606 & 17.68 \\
\hline 6 & 48.23 & 18.896 & 13.92 \\
\hline 5 & 38.21 & 14.322 & 10.31 \\
\hline 4 & 27.9 & 10.039 & 6.993 \\
\hline 3 & 17.66 & 6.2201 & 4.107 \\
\hline 2 & 8.18 & 3.0267 & 1.819 \\
\hline 1 & 1.179 & 0.6304 & 0.295 \\
\hline 0 & 0 & 0 & 0 \\
\hline
\end{tabular}




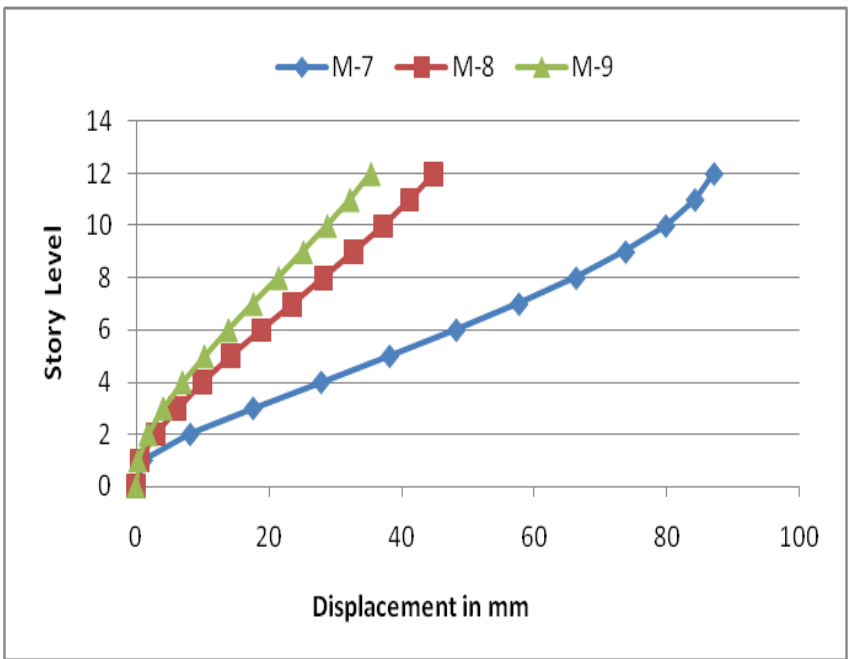

Fig 15: Lateral displacement in Transverse direction

Table 8: Lateral displacement in longitudinal direction (RSA)

\begin{tabular}{|l|l|l|l|}
\hline \multicolumn{4}{|l|}{ Stepback buildings } \\
\hline Story & M-1 & M-2 & M-3 \\
\hline 12 & 27.745 & 20.028 & 15.521 \\
\hline 11 & 26.518 & 18.96 & 13.887 \\
\hline 10 & 24.767 & 17.529 & 12.166 \\
\hline 9 & 22.415 & 15.734 & 10.392 \\
\hline 8 & 19.49 & 13.621 & 8.5906 \\
\hline 7 & 16.052 & 11.245 & 6.8003 \\
\hline 6 & 12.188 & 8.6581 & 5.0681 \\
\hline 5 & 8.0701 & 5.9306 & 3.4491 \\
\hline 4 & 4.0658 & 3.2061 & 2.0087 \\
\hline 3 & 0.9547 & 0.8975 & 0.8055 \\
\hline 2 & 0 & 0 & 0 \\
\hline 1 & 0 & 0 & 0 \\
\hline
\end{tabular}

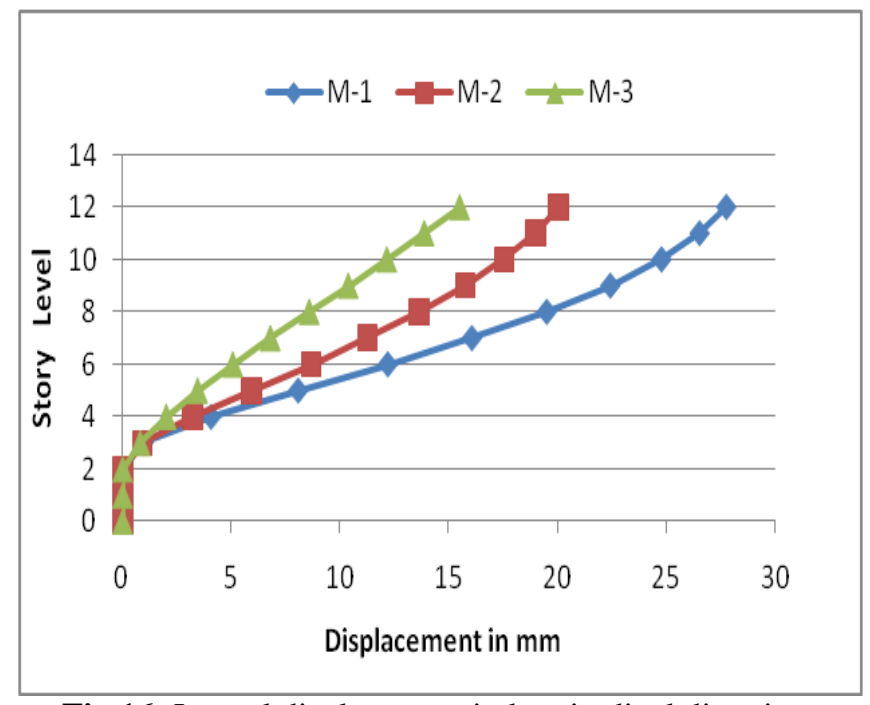

Fig 16: Lateral displacement in longitudinal direction
Table 9: Lateral displacement in Transverse direction (RSA)

\begin{tabular}{|c|r|r|r|}
\hline \multicolumn{4}{|c|}{ StepBack } \\
\hline Story & M-1 & M-2 & \multicolumn{1}{c|}{ M-3 } \\
\hline 12 & 21.24 & 16.413 & \multicolumn{1}{c|}{12.61} \\
\hline 11 & 20.44 & 15.499 & 11.29 \\
\hline 10 & 19.19 & 14.265 & 9.857 \\
\hline 9 & 17.46 & 12.743 & 8.35 \\
\hline 8 & 15.28 & 10.983 & 6.792 \\
\hline 7 & 12.7 & 9.0336 & 5.227 \\
\hline 6 & 9.774 & 6.9409 & 3.713 \\
\hline 5 & 6.589 & 4.7553 & 2.322 \\
\hline 4 & 3.378 & 2.5614 & 1.141 \\
\hline 3 & 0.77 & 0.6585 & 0.279 \\
\hline 2 & 0 & 0 & 0 \\
\hline 1 & 0 & 0 & 0 \\
\hline 0 & 0 & 0 & 0 \\
\hline
\end{tabular}

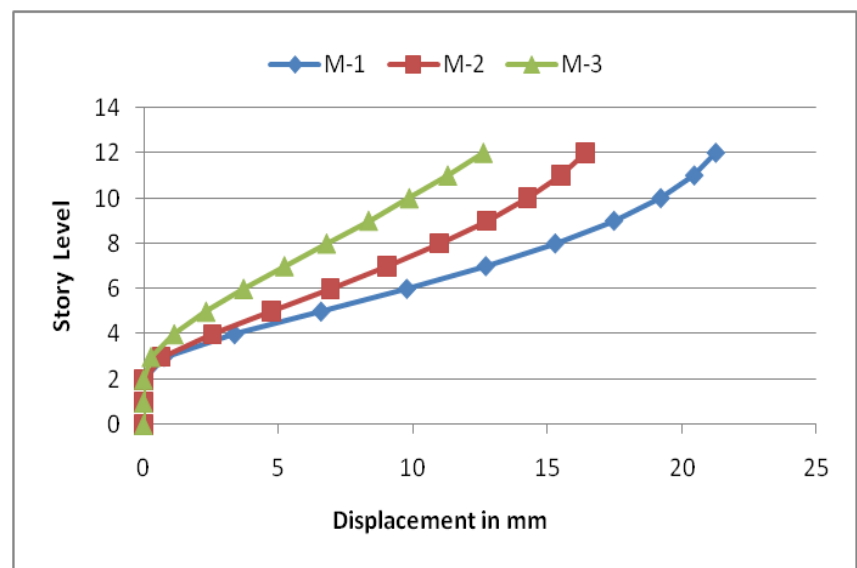

Fig 17: Lateral displacement in Transverse direction

Table 10: Lateral displacement in Longitudinal direction (RSA)

\begin{tabular}{|c|r|r|r|}
\hline \multicolumn{4}{|c|}{ SetbckStepbck buildings } \\
\hline Story & M-4 & \multicolumn{1}{|c|}{ M-5 } & \multicolumn{1}{c|}{ M-6 } \\
\hline 12 & 26.415 & 19.246 & 15.096 \\
\hline 11 & 25.169 & 18.22 & 13.679 \\
\hline 10 & 23.552 & 16.927 & 12.01 \\
\hline 9 & 21.447 & 15.339 & 10.297 \\
\hline 8 & 18.784 & 13.402 & 8.5417 \\
\hline 7 & 15.565 & 11.145 & 6.7846 \\
\hline 6 & 11.872 & 8.6272 & 5.0722 \\
\hline 5 & 7.8853 & 5.9318 & 3.4619 \\
\hline 4 & 3.9809 & 3.2152 & 2.0216 \\
\hline 3 & 0.9363 & 0.9022 & 0.8131 \\
\hline 2 & 0 & 0 & 0 \\
\hline 1 & 0 & 0 & 0 \\
\hline 0 & 0 & 0 & 0 \\
\hline
\end{tabular}




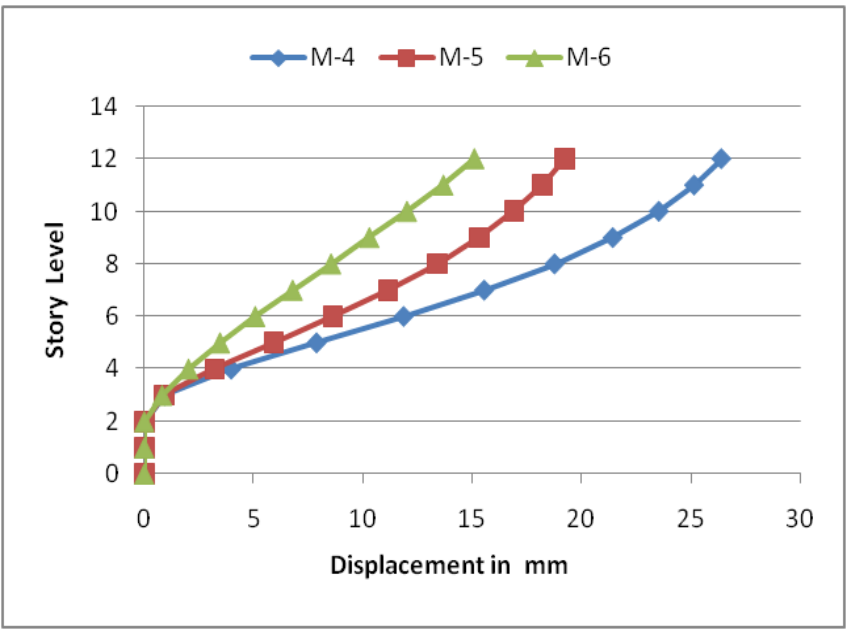

Fig 18: Lateral displacement in longitudinal direction

Table 11: Lateral displacement in Transverse direction (RSA)

\begin{tabular}{|c|r|r|r|}
\hline \multicolumn{5}{|c|}{ SetBackStepBack } \\
\hline Story & M-4 & M-5 & M-6 \\
\hline 12 & 27.52 & 19.181 & 14.65 \\
\hline 11 & 26.58 & 18.19 & 13.13 \\
\hline 10 & 25.07 & 16.861 & 11.53 \\
\hline 9 & 22.9 & 15.166 & 9.798 \\
\hline 8 & 20.1 & 13.142 & 7.993 \\
\hline 7 & 16.72 & 10.849 & 6.166 \\
\hline 6 & 12.85 & 8.3484 & 4.386 \\
\hline 5 & 8.611 & 5.7123 & 2.743 \\
\hline 4 & 4.344 & 3.0555 & 1.343 \\
\hline 3 & 0.911 & 0.7538 & 0.323 \\
\hline 2 & 0 & 0 & 0 \\
\hline 1 & 0 & 0 & 0 \\
\hline 0 & 0 & 0 & 0 \\
\hline
\end{tabular}

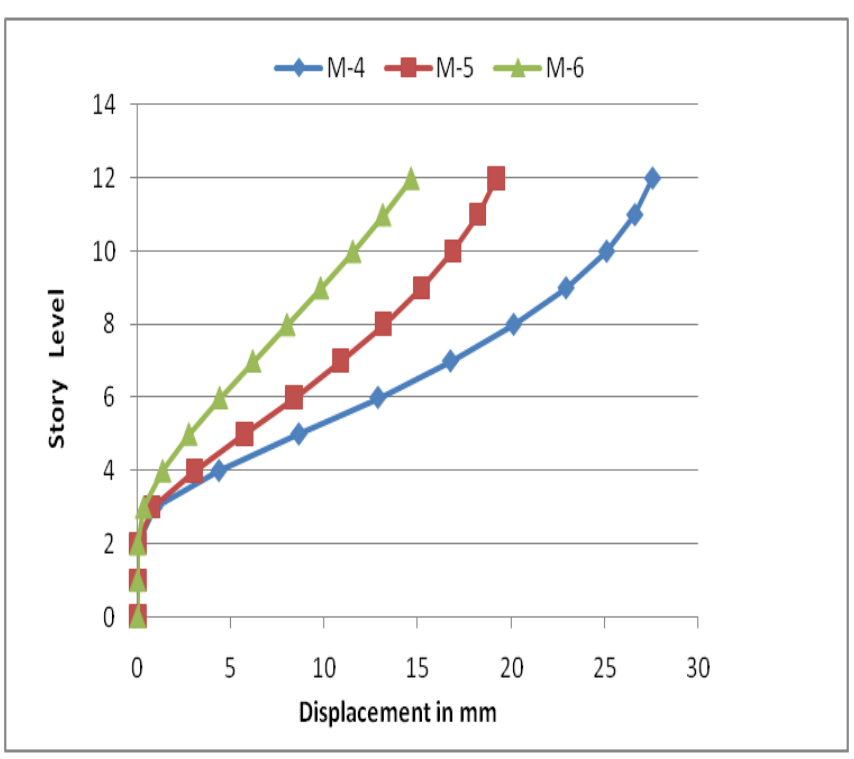

Fig 19: Lateral displacement in longitudinal direction
Table12: Lateral displacement in Longitudinal direction (RSA)

\begin{tabular}{|c|r|r|r|}
\hline \multicolumn{4}{|c|}{ Plain Ground } \\
\hline Story & M-7 & M-8 & \multicolumn{1}{c|}{ M-9 } \\
\hline 12 & 33.786 & 21.043 & 17.575 \\
\hline 11 & 32.7 & 19.398 & 15.885 \\
\hline 10 & 31.156 & 17.569 & 14.11 \\
\hline 9 & 29.077 & 15.614 & 12.282 \\
\hline 8 & 26.481 & 13.567 & 10.421 \\
\hline 7 & 23.407 & 11.465 & 8.5612 \\
\hline 6 & 19.893 & 9.3479 & 6.7414 \\
\hline 5 & 15.985 & 7.2614 & 5.0095 \\
\hline 4 & 11.765 & 5.2555 & 3.4206 \\
\hline 3 & 7.4215 & 3.3892 & 2.0376 \\
\hline 2 & 3.3673 & 1.718 & 0.9281 \\
\hline 1 & 0.4762 & 0.3446 & 0.1523 \\
\hline 0 & 0 & 0 & 0 \\
\hline
\end{tabular}

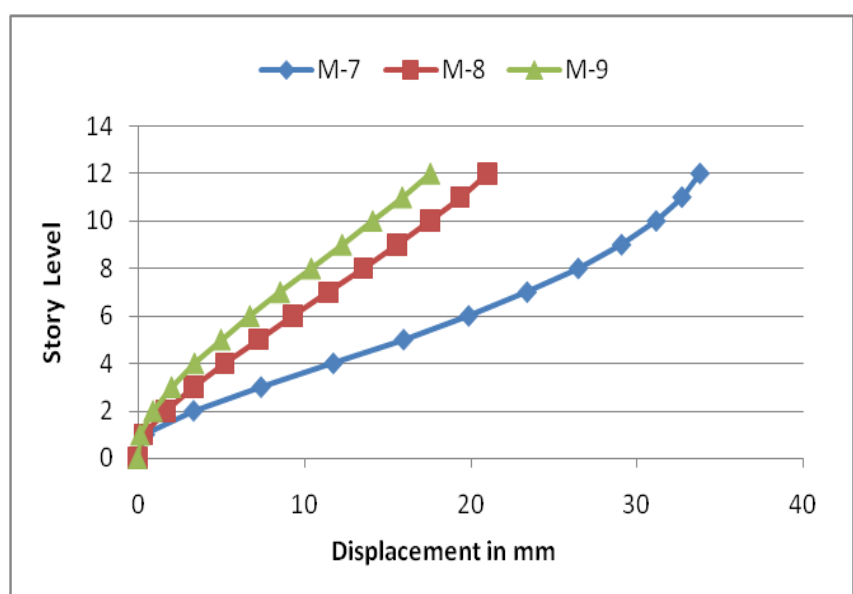

Fig 20: Lateral displacement in longitudinal direction

Table 13: Lateral displacement in Transverse direction (RSA)

\begin{tabular}{|c|r|r|r|}
\hline \multicolumn{4}{|c|}{ Plain Ground } \\
\hline Story & M-7 & M-8 & M-9 \\
\hline 12 & 30.99 & 22.903 & 20.31 \\
\hline 11 & 30.1 & 21.119 & 18.53 \\
\hline 10 & 28.76 & 19.134 & 16.63 \\
\hline 9 & 26.91 & 17.014 & 14.63 \\
\hline 8 & 24.59 & 14.784 & 12.55 \\
\hline 7 & 21.86 & 12.48 & 10.42 \\
\hline 6 & 18.74 & 10.147 & 8.295 \\
\hline 5 & 15.27 & 7.838 & 6.226 \\
\hline 4 & 11.47 & 5.6195 & 4.287 \\
\hline 3 & 7.462 & 3.5754 & 2.566 \\
\hline 2 & 3.535 & 1.7948 & 1.164 \\
\hline 1 & 0.517 & 0.3896 & 0.195 \\
\hline 0 & 0 & 0 & 0 \\
\hline
\end{tabular}




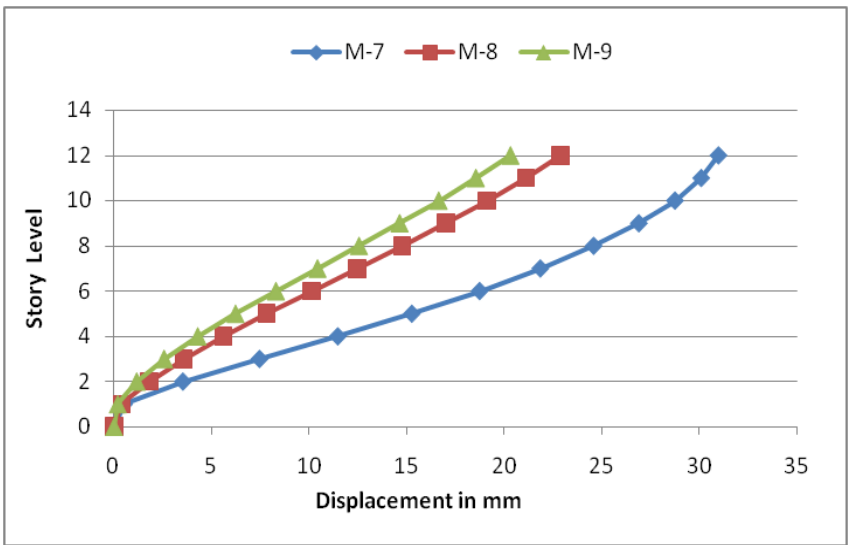

The Performance point of the building models in longitudinal \& transverse direction are tabulated in table 15 to table 17 as obtained from ETABS.

Fig 21: Lateral displacement in longitudinal direction

\subsection{Performance Point}

Table 14: The values of seismic coefficient $\mathrm{Ca}$ and $\mathrm{Cv}$ for Zone $\mathrm{V}$ are Taken from below Table Seismic Coefficients: $\mathrm{Ca}$

\begin{tabular}{|l|l|l|l|l|}
\hline Soil & Zone II $(0.1)$ & Zone III $(0.16)$ & Zone IV $(0.24)$ & Zone $(0.36)$ \\
\hline Type I & 0.12 & 0.19 & 0.28 & 0.37 \\
\hline Type II & 0.15 & 0.23 & 0.31 & $\mathbf{0 . 4 1}$ \\
\hline Type III & 0.23 & 0.31 & 0.35 & 0.36 \\
\hline Seismic Coefficients: Cv & 0.26 & 0.37 & 0.52 \\
\hline Type I & 0.17 & 0.34 & 0.46 & $\mathbf{0 . 6}$ \\
\hline Type II & 0.23 & 0.3 & 0.72 & 0.91 \\
\hline Type III & 0.34 & &
\end{tabular}

Table 15: Performance parameter for Set1 (Step Back Buildings) along longitudinal \& Transverse Direction

\begin{tabular}{|l|l|l|l|l|l|l|l|l|}
\hline & \multicolumn{6}{|l|}{ Transverse } & \multicolumn{2}{l|}{ Roof } \\
\hline Model & $\begin{array}{l}\text { Base } \\
\text { shear } \\
\mathrm{V}(\mathrm{KN})\end{array}$ & $\begin{array}{l}\text { Roof } \\
\text { Displacemen } \\
\mathrm{t}(\mathrm{D})\end{array}$ & $\begin{array}{l}\text { Structural } \\
\text { acceleration(Sa) }\end{array}$ & $\begin{array}{l}\text { Structural } \\
\text { Displacem } \\
\text { ent(Sd) }\end{array}$ & $\begin{array}{l}\text { Base } \\
\text { shear } \\
\text { V(KN) }\end{array}$ & $\begin{array}{l}\text { Displacemen } \\
\mathrm{t}(\mathrm{D})\end{array}$ & $\begin{array}{l}\text { Structural } \\
\text { acceleratio } \\
\mathrm{n}(\mathrm{Sa})\end{array}$ & $\begin{array}{l}\text { Structural } \\
\text { Displacem } \\
\text { ent(Sd) }\end{array}$ \\
\hline 1 & - & - & - & - & - & - & - & - \\
\hline 2 & $\begin{array}{l}2333.9 \\
74\end{array}$ & 253.842 & 0.326 & 173.76 & - & - & - & - \\
\hline 3 & $\begin{array}{l}11149 . \\
618\end{array}$ & 237 & 0.316 & 155.9 & - & - & - & - \\
\hline
\end{tabular}

Table 16: Performance parameter for Set2 (SetBackStepBack) along longitudinal \& Transverse Direction

\begin{tabular}{|l|l|l|l|l|l|l|l|l|}
\hline & \multicolumn{9}{l|}{ Transverse } \\
\hline $\begin{array}{l}\text { Mode } \\
1\end{array}$ & $\begin{array}{l}\text { Base } \\
\text { shear } \\
\mathrm{V}(\mathrm{KN})\end{array}$ & $\begin{array}{l}\text { Roof } \\
\text { Displacemen } \\
\mathrm{t}(\mathrm{D})\end{array}$ & $\begin{array}{l}\text { Structural } \\
\text { acceleration(Sa) }\end{array}$ & $\begin{array}{l}\text { Structural } \\
\text { Displacem } \\
\text { ent(Sd) }\end{array}$ & $\begin{array}{l}\text { Base } \\
\text { shear } \\
\text { V(KN) }\end{array}$ & $\begin{array}{l}\text { Roof } \\
\text { Displacemen } \\
\mathrm{t}(\mathrm{D})\end{array}$ & $\begin{array}{l}\text { Structural } \\
\text { acceleratio } \\
\mathrm{n}(\mathrm{Sa})\end{array}$ & $\begin{array}{l}\text { Structural } \\
\text { Displacem } \\
\text { ent(Sd) }\end{array}$ \\
\hline 1 & - & - & - & - & - & - & - & - \\
\hline 2 & 12321.3 & 277.128 & 0.374 & 181.173 & $\begin{array}{l}10742.6 \\
87\end{array}$ & 309.5 & 0.323 & 199.48 \\
\hline 3 & 10227.0 & 220 & 0.317 & 133 & - & - & - & - \\
\hline
\end{tabular}

Table 17: Performance parameter for Set3 (Plain Ground) along longitudinal \& Transverse Direction

\begin{tabular}{|l|l|l|l|l|l|l|l|l|}
\hline & \multicolumn{6}{|l|}{ Longitudinal } \\
\hline Model & $\begin{array}{l}\text { Base } \\
\text { shear } \\
\mathrm{V}(\mathrm{KN})\end{array}$ & $\begin{array}{l}\text { Roof } \\
\text { Displacemen } \\
\mathrm{t}(\mathrm{D})\end{array}$ & $\begin{array}{l}\text { Structural } \\
\text { acceleration(Sa) }\end{array}$ & $\begin{array}{l}\text { Structural } \\
\text { Displacem } \\
\text { ent(Sd) }\end{array}$ & $\begin{array}{l}\text { Base } \\
\text { shear } \\
\text { V(KN) }\end{array}$ & $\begin{array}{l}\text { Roof Displacemen } \\
\text { t(D) }\end{array}$ & $\begin{array}{l}\text { Structural } \\
\text { acceleratio } \\
\mathrm{n}(\mathrm{Sa})\end{array}$ & $\begin{array}{l}\text { Structural } \\
\text { Displacem } \\
\text { ent }(\mathrm{Sd})\end{array}$ \\
\hline 1 & 3483.69 & 518 & 0.076 & 377 & 3206.267 & 549.866 & 0.071 & 391.197 \\
\hline 2 & - & - & - & - & - & - & - & - \\
\hline 3 & 10961.2 & 262.343 & 0.296 & 171.538 & 7214.36 & 347.713 & 0.195 & 227.640 \\
\hline
\end{tabular}


From the above table 15 to table 17 it is observed that that the Model M-1 is nil indicating the capacity is less than demand, such structures will not perform well where as Model M-2,M-3 show the performance point in longitudinal direction \& In Transverse direction the Model M-1,M-2,M-3 is nil.

For SetbackStepback Buildings Models M-4 is Nil, Model M-5 ,M-6 shows the performance point the Structural displacement \& Roof displacement is decreasing respectively in longitudinal direction \& in transverse direction the Model M-5 shows the performance point where as M-4 \&M-6 is nil.

For buildings on Plain Ground the model M-8 is Nil \& Model M-7 ,M-9 show the performance point .the structural displacement \& Roof displacement is found decreasing ,where as Structural acceleration \& shear force is increasing respectively in Longitudinal direction \& transverse direction.

From the above we can say that influence of shear Wall reduce the Structural displacements \& Roof displacement

\subsection{Design}

From the above Results and discussion of Fundamental time period \& Lateral displacement the Model M-6 SetbackStepback is found to be superior on sloping ground compared StepBack Buildings. The design is carried out with aid of IS456 and IS 13920:1993

General specification

The member is designed according to IS 456:2000

Building > 3 storey height, minimum grade of concrete M20

- we used M25

Steel reinforcement of FE 415 used.

1. Design of Flexural Member

2. Design of Column

\subsubsection{Design of Flexural Member}

\subsubsection{General}

1) Factored axial stress less than $0.1 \mathrm{f}_{\mathrm{ck}}$

2) The member should preferably have a width to depth ratio of more 0.3

Width/depth $=300 / 600=0.5>0.3$, hence ok

3 ) Width should not be less then $200 \mathrm{~mm}$. But we provided width of $300 \mathrm{~mm}$ which is ok.

4) Depth should not be greater than 0.25 (clear span) i.e. $(7000-300)=1675 \mathrm{~mm}$.

\subsubsection{Longitudinal Reinforcement}

At End

Top Rebar $\%=0.68$

Bottom Rebar \% $=0.40$

Steel provided @ Top 2\#16 mm dia + 3\# 20 mm dia.

Steel Provided @ Bottom 3 \#16 mm dia
Through 16 dia are provided @ top \& Bottom

At mid Span

Top Rebar $\%=0.29$

Bottom \%=0.38

Steel provided @Top_2\#20dia

Steel provided @Bottom 3\#16 dia

\subsubsection{Shear Reinforcement}

Vu $163.41 \mathrm{KN}$

Stirrups 8 dia@ $75 \mathrm{~mm} \mathrm{c/c}$ from face of column upto 1130mm\&8dia@250 mm c/c at mid span

\subsubsection{Design of Column}

We have size of the column $700 \mathrm{~mm} \times 850 \mathrm{~mm}$

Concrete mix M 25

Vertical reinforcement Fe 415

Axial load $2464 \mathrm{KN}$

\subsubsection{General (Column Subjected to Bending and} Axial Load)

IS 13920:193 will be applicable if the axial stress $>0.1 \mathrm{f}_{\mathrm{ck}}$.

$2464 \times 1000 /(700 \times 850)=4.14>0.1 \mathrm{f}_{\mathrm{ck}}=2.5$

Minimum dimension of the member $\geq 250$ and we have taken 400 which is ok.

Shortest cross section dimension / perpendicular dimension $\geq 0.4$ and we have the same ratio as 0.82

\subsubsection{Vertical Reinforcement}

6 - \# 32 dia (3 at each face)

\subsubsection{Shear Reinforcement}

$8 \mathrm{~mm}$ dia 2-LVS about $125 \mathrm{~mm} \mathrm{c/c}$

Special confining reinforcement is to be provided over a length of lo towards the mid span of column

$\mathrm{L} 0 \geq$ depth of the member $=850 \mathrm{~mm}$

$[1 / 6$ of the clear span which is $500 \mathrm{~mm}$ in this case] [450 $\mathrm{mm}$ ]

The spacing of the hoop shall not exceed

$\mathrm{S} \max <1 / 4($ minimum member dimensions $)=175 \mathrm{~mm}$ in this case]

Should not be less than 75]

Should not be greater than 100]

Minimum area of cross section of the bar forming hoop is

$\mathrm{A}_{\mathrm{sh}}=0.18 \mathrm{Sh} f_{c k} / f_{y}\left(\mathrm{~A}_{\mathrm{g}} / \mathrm{A}_{\mathrm{k}}-1\right)$

We use $S=10 \mathrm{~mm}$ from above and $\mathrm{h}=395$

So we have $A_{\mathrm{sh}}=46.25 \mathrm{~mm}^{2}$. 
Using $10 \mathrm{~mm}$ dia bar $\left(78.53 \mathrm{~mm}^{2}\right)$ at a spacing of $100 \mathrm{x}$ $78.53 / 46.25=81.56 \mathrm{~mm}$ i.e @ $80 \mathrm{~mm} \mathrm{c/c}$

\section{DUCTILITY DETAILING}

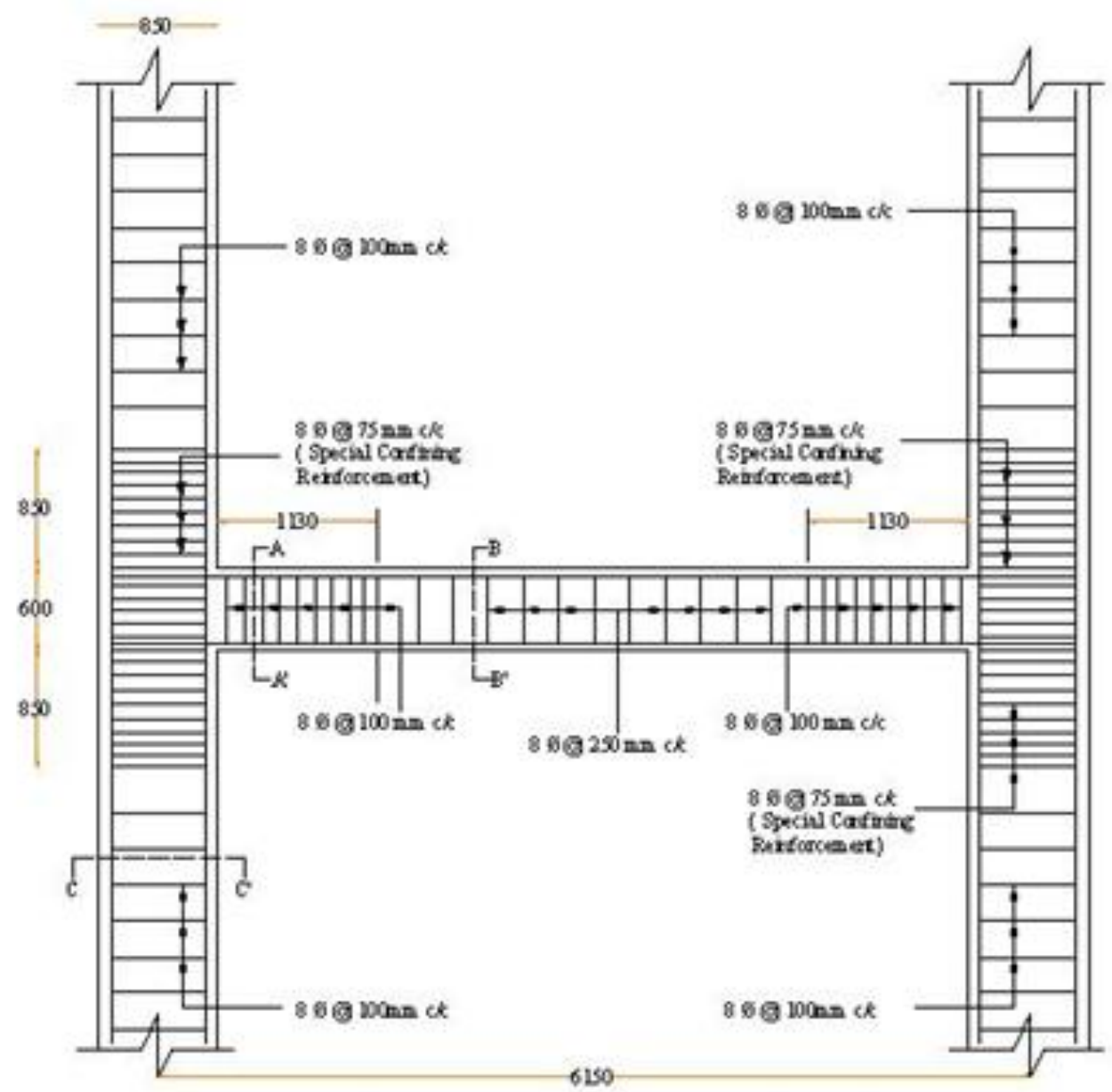

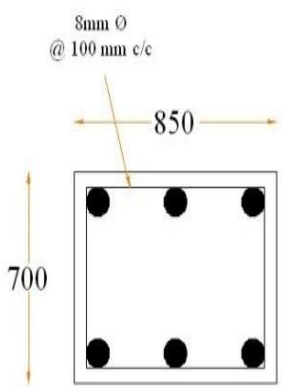

$6-\# 320$

AT CC'

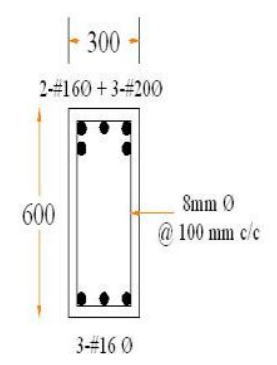

ATAA'

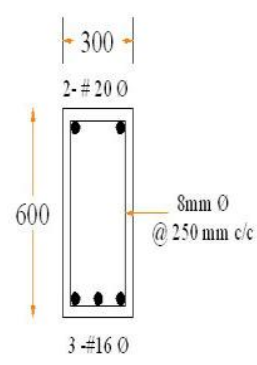

$\mathrm{ATBB}^{\prime}$ 


\section{CONCLUSIONS}

1. Fundamental natural period decreases when effect of bracings and concrete shear wall is considered

2. Displacements are found to within the limit in linear static method, linear dynamic and non-linear static analysis.

3. The Study gave an idea to study the maximum stiffness with bracings, shear wall.

4. The presence bracings, shear wall influences the overall behavior of structures when subjected to lateral forces. Joint displacements and story drifts are considerably reduced.

5. Results indicate that shear wall have large effect on the behavior of frames under earthquake excitation

6. From the results it is observed that shear wall effect stiffness of the frame, due to which comparatively less reinforcement is required as compared to reinforcement required in bare frame, bracing frame.

7. The SetbackStepback on Sloping ground possesses relatively less displacements when compared to StepBack buildings on Sloping ground \& Plain Ground

8. The performance of the buildings on sloping ground suggests an increased vulnerability of the structure with formation of column hinges at base level and beam hinges at each story level at performance point.

\section{REFERENCES}

[1] Birajdar \& S.S.Nalawade. "Seismic Analysis Of Buildings Resting On Sloping Ground B.G"

[2] Dr. Sanjaya Kumar Patro, Susanta Banerjee, Debasnana Jena, Sourav Kumar Das. "A Review on Seismic Analysis Of a Building on sloping ground".

[3] S.M.Nagargoje and K.S. Sable , "Seismic performance of multi-storeyed Building on Sloping Ground.

[4] M.D. Kevadkar, P.B Kodag "Lateral load analysis on R.C.C Building".

[5] Alfa Rasikan , M.G.Rajendran "Wind behavior of buildings with and without shear wall".

[6] P. S. Kumbhare, A. C. Saoji "Effectiveness of Changing Reinforced Concrete Shear Wall Location on Multi-storied Building".

[7] IS1893,"Criteria for Earthquake Resistant Design of Structures (part 1) General Provision and Buildings(Fifth Revision)",Bureau of Indian Standards, 2002.

[8] IS 456, "Plain and Reinforced Concrete-Code of Practice", Bureau of Indian Standards, 2000.

[9] IS 13920 , "Ductile Detailing of Reinforced Concrete Structures Subjected to Seismic Forces Code of Practice", Bureau of Indian Standards, 1993.

[10] SP16, "Design Aids for Reinforced Concrete", Bureau of Indian Standards, 1993.

[11] IITK Earthquake tip22. 